\title{
Hanging Lesion Maneuver: Superficial Shave Excision of Benign Lesion Limited Cutis with The Simple Technique
}

\author{
Murat CAKIR ${ }^{1}$, Muslim Yurtcu ${ }^{2}$, Ahmet Tekin ${ }^{3}$, Tevfik Kucukkartallar ${ }^{4}$ \\ And Adil Kartal ${ }^{5}$ \\ 1,3,4,5, Department of General Surgery Meram Medical Faculty, Necmettin Erbakan University, \\ , Konya, Turkey \\ ${ }^{2}$ Departments of Pediatric Surgery, Meram Medical Faculty, Necmettin Erbakan University, \\ Konya, Turkey
}

Correspondence should be addressed to: Murat CAKIR; drmuratcakir@hotmail.com

Received date: 24 June 2014; Accepted date: 28 september 2014; Published date: 8 september 2016

Copyright (C) 2016. Murat CAKIR, Muslim Yurtcu, Ahmet Tekin, Tevfik Kucukkartallar And Adil Kartal. Distributed under Creative Commons CC-BY 4.0

\begin{abstract}
It is one of the most important problems for surgeons to decide which operation should be undertaken on children with benign superficial lesion, especially those with benign mini lesions. Recently, removal techniques using shave excision in order to make surgical treatment have become very popular for patients with benign superficial mini lesion limited cutis. We used hanging lesion maneuver instead of shave excision to treat benign superficial mini lesions. We conclude that hanging mini lesion maneuver performed under sterile conditions may be an alternative to conventional shave excision in daily practice, for preventing excessive scarring and discoloration in children undergoing benign superficial mini lesion surgery.
\end{abstract}

Keywords: superficial lesion, benign, shave excision

\section{Introduction}

There are some important indications in mini lesions (MLs), such as benign melanocytic lesions and superficial nevi. Shave excision, which is performed to treat these kind of lesions (figure 1), is frequently applied; but, the possibility of recurrence and the actual cosmetic results achieved with this procedure (shave excision) remains a controversial issue (Ferrandız, 2005; Bong et al., 2003; Gambichler et al., 2000; Breuninger et al., 2000).

Cite this Article as: Murat CAKIR, Muslim Yurtcu, Ahmet Tekin, Tevfik Kucukkartallar And Adil Kartal (2016), "Hanging Lesion Maneuver: Superficial Shave Excision of Benign Lesion Limited Cutis with The Simple Technique ", JMED Research, Vol. 2016 (2016), Article ID 975040, DOI: 10.5171/2016.975040 


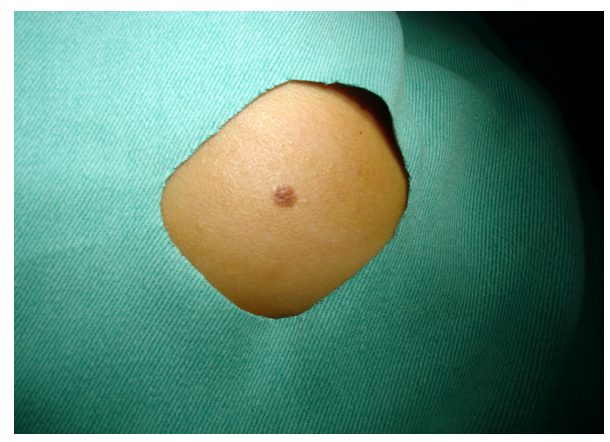

Figure 1: Appearance of the mini lesion

We consider the use of hanging lesion maneuver (HLM) as more practical and scientific technique when shave excision is not possible. In shave excision procedure, mini spesimens (MSs) may lose, because it is smaller than normal size and histopathological manipulation is not as easy as large spesimen.

To the best of our knowledge, this is the first technique in which the removal of MSs will be excised for this purpose.

\section{Technique}

The HLM technique was performed under sterile conditions. Under anesthesia with sublesional (dermis, internal dermis, and subcutaneous) $2 \%$ mepivacaine with insuline injection needle, the HLM was performed using nonabsorbable suture material (6-0 polypropylene) of which two ends are with needles for the superficial shave excision of benign MLs limited cutis. The suture material was kept with 1-2 fingers and the lesion was hung after passing from the middle and just margin of the lesion (Figure 2). The ML hung was incised horizontally with a number 15 surgical blade from one or both sides without changing incision platform. The specimens (with suture) obtained were fixed in a tube of $10 \%$ formaldehyde for histopathologic examination and was sent to the department of pathology (Figure 3). All MSs were examined by a pathologist, while the ML was hanging in the suture material. There was no risk of loss of the specimen accidentally, because it was hung with the suture material (Figure 3). This suture material may not be used in the superficial MLs which are limited to only the epidermis and dermis. The biopsy areas which were settled in the deep dermis were usually closed with 6-0 polypropylene or left open. The suture material was removed in early period and the area recovered without an obvious scarring

(Figure

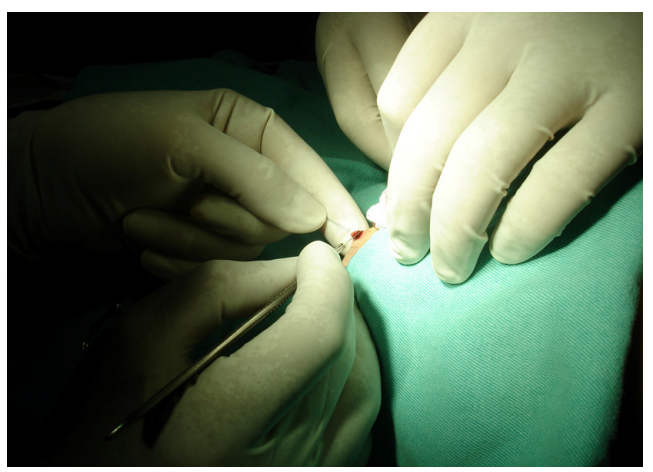

Figure 2: Suture material passing from the middle of lesion. 


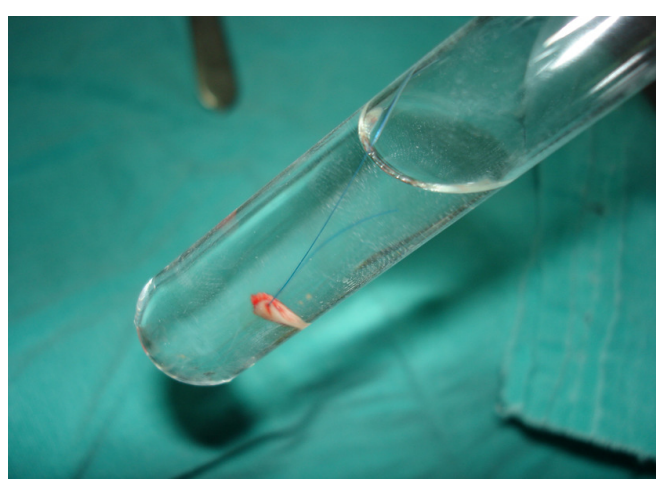

Figure 3: Appearance of the mini specimen fixed by suture material in the tube for histopathological examination.

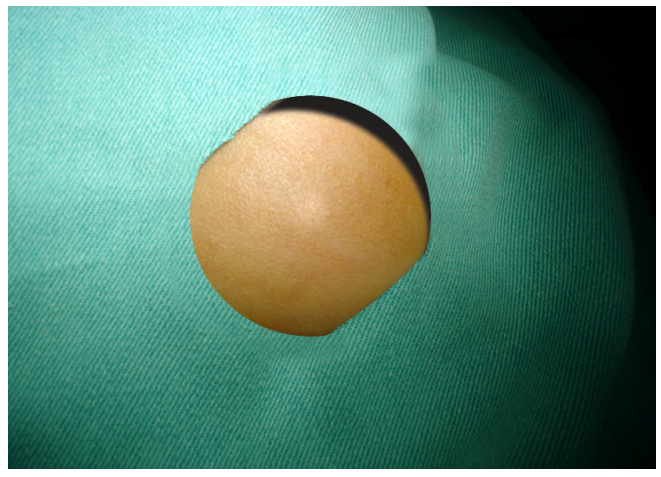

Figure 4: Appearance of the healed wound tissue after removal of mini lesion in postoperative period.

\section{Discussion}

In studies concerning melanocytic nevi, excisions were based on shave excision (Ferrandız, 2005; Bong et al., 2003; Gambichler et al., 2000; Breuninger et al., 2000). Gambichler et al (2000) suggested that deep shave excision of macular melanocytic naevi with the razor blade is a good technique. In addition, Ferrandız et al (2005) reported that shave excision is endicated for common acquired melanocytic nevi. However, these techniques are controversial because the risk of cosmetic result is presumed. A common undesirable feature of these techniques is that their scarring is present.

In the HLM technique described here, a small incision is done, the suture material for repair is not always used, there was not generally residual tissue, and the procedure is repeated in the cases with waste tissue. The same suture material is used in both hanging and suturing the biopsy area. Lesions, which were larger than $5 \mathrm{~mm}$, were excised with double suture materials to balance the lesion. The MS kept in the suture material is preserved in the sure conditions even if the tube is broken accidentally, and is seen in the tube easily in this technique. Thus, sufficient and whole materials are sent to the department of pathology. The same pathologist takes both the MS from the tube after tracting the suture material easily.

We conclude that HLM performed under sterile conditions may be an alternative to shave excision in daily practice, for 
preventing scarring and discoloration in patients with benign superficial MLs. The HLM is simple, fast, efficient, and of low cost and low risk for the patient. The results depend on the experience and ability of the surgeon.

\section{Conflict of Interest Disclosure Statement}

Murat Cakir and other co-authors have no conflict of interest.

\section{References}

1. Bong, JL. \& Perkins, W., (2003). 'Shave excision of benign facial melanocytic naevi: a patient's satisfaction survey' Dermatol Surg, 29, 227-229.
2. Breuninger, H., Garbe, C. \& Rassner, G, (2000). ,Shave excision von melanozytaren Navi der Haut', Hautarzt, 51, 575-580.

3. Ferrandız, L., (2005). 'Shave excision of common acquired melanocytic nevi: cosmetic outcome, recurrences, and complications' Dermatol Surg, 31, 11121115.

4. Gambichler, T., Senger, E., Rapp, S., Alamouti, D., Altmeyer, P. \& Hoffmann, K, (2000). 'Deep shave excision of macular melanocytic naevi with the razor blade biopsy technique' Dermatol Surg, 26, 662666. 